\title{
Magnetic flux concentrations in a polytropic atmosphere
}

\author{
I. R. Losada ${ }^{1,2}$, A. Brandenburg ${ }^{1,2}$, N. Kleeorin ${ }^{3,1,4}$, and I. Rogachevskii ${ }^{3,1,4}$
}

\author{
1 Nordita, KTH Royal Institute of Technology and Stockholm University, Roslagstullsbacken 23, 10691 Stockholm, Sweden \\ e-mail: illa.rivero.losada@gmail.com \\ 2 Department of Astronomy, AlbaNova University Center, Stockholm University, 10691 Stockholm, Sweden \\ 3 Department of Mechanical Engineering, Ben-Gurion University of the Negev, POB 653, 84105 Beer-Sheva, Israel \\ ${ }^{4}$ Department of Radio Physics, N. I. Lobachevsky State University of Nizhny Novgorod, 603950 Nizhnü Novgorod, Russia
}

Received 18 July 2013 / Accepted 23 January 2014

\section{ABSTRACT}

\begin{abstract}
Context. Strongly stratified hydromagnetic turbulence has recently been identified as a candidate for explaining the spontaneous formation of magnetic flux concentrations by the negative effective magnetic pressure instability (NEMPI). Much of this work has been done for isothermal layers, in which the density scale height is constant throughout.

Aims. We now want to know whether earlier conclusions regarding the size of magnetic structures and their growth rates carry over to the case of polytropic layers, in which the scale height decreases sharply as one approaches the surface.

Methods. To allow for a continuous transition from isothermal to polytropic layers, we employ a generalization of the exponential function known as the $q$-exponential. This implies that the top of the polytropic layer shifts with changing polytropic index such that the scale height is always the same at some reference height. We used both mean-field simulations (MFS) and direct numerical simulations (DNS) of forced stratified turbulence to determine the resulting flux concentrations in polytropic layers. Cases of both horizontal and vertical applied magnetic fields were considered.

Results. Magnetic structures begin to form at a depth where the magnetic field strength is a small fraction of the local equipartition field strength with respect to the turbulent kinetic energy. Unlike the isothermal case where stronger fields can give rise to magnetic flux concentrations at larger depths, in the polytropic case the growth rate of NEMPI decreases for structures deeper down. Moreover, the structures that form higher up have a smaller horizontal scale of about four times their local depth. For vertical fields, magnetic structures of super-equipartition strengths are formed, because such fields survive downward advection that causes NEMPI with horizontal magnetic fields to reach premature nonlinear saturation by what is called the "potato-sack" effect. The horizontal cross-section of such structures found in DNS is approximately circular, which is reproduced with MFS of NEMPI using a vertical magnetic field. Conclusions. Results based on isothermal models can be applied locally to polytropic layers. For vertical fields, magnetic flux concentrations of super-equipartition strengths form, which supports suggestions that sunspot formation might be a shallow phenomenon.
\end{abstract}

Key words. magnetohydrodynamics (MHD) - hydrodynamics - turbulence - Sun: dynamo

\section{Introduction}

In a turbulent medium, the turbulent pressure can lead to dynamically important effects. In particular, a stratified layer can attain a density distribution that is significantly altered compared to the nonturbulent case. In addition, magnetic fields can change the situation further, because it can locally suppress the turbulence and thus reduce the total turbulent pressure (the sum of hydrodynamic and magnetic turbulent contributions). On length scales encompassing many turbulent eddies, this total turbulent pressure reduction must be compensated for by additional gas pressure, which can lead to a density enhancement and thus to horizontal magnetic structures that become heavier than the surroundings and sink (Brandenburg et al. 2011). This is quite the contrary of magnetic buoyancy, which is still expected to operate on the smaller scale of magnetic flux tubes and in the absence of turbulence. Both effects can lead to instability: the latter is the magnetic buoyancy or interchange instability (Newcomb 1961; Parker 1966), and the former is now often referred to as negative effective magnetic pressure instability (NEMPI), which has been studied at the level of mean-field theory for the past two decades (Kleeorin et al. 1989, 1990, 1993, 1996; Kleeorin \& Rogachevskii 1994; Rogachevskii \& Kleeorin 2007). These are instabilities of a stratified continuous magnetic field, while the usual magnetic buoyancy instability requires nonuniform and initially separated horizontal magnetic flux tubes (Parker 1955; Spruit 1981; Schüssler et al. 1994).

Unlike the magnetic buoyancy instability, NEMPI occurs at the expense of turbulent energy rather than the energy of the gravitational field. The latter is the energy source of the magnetic buoyancy or interchange instability. NEMPI is caused by a negative turbulent contribution to the effective mean magnetic pressure (the sum of nonturbulent and turbulent contributions). For large Reynolds numbers, this turbulent contribution to the effective magnetic pressure is larger than the nonturbulent one. This results in the excitation of NEMPI and the formation of large-scale magnetic structures - even from an originally uniform mean magnetic field.

Direct numerical simulations (DNS) have recently demonstrated the operation of NEMPI in isothermally stratified layers (Brandenburg et al. 2011; Kemel et al. 2012b). This is a particularly simple case in that the density scale height is constant; i.e., the computational burden of covering large density variation is distributed over the depth of the entire layer. In spite of this simplification, it has been argued that NEMPI is important for explaining prominent features in the manifestation of solar surface activity. In particular, it has been associated with the formation of active regions (Kemel et al. 2013; Warnecke et al. 2013) and sunspots (Brandenburg et al. 2013, 2014). However, it is now important to examine the validity of conclusions based on 
such simplifications using more realistic models. In this paper, we therefore now consider a polytropic stratification, for which the density scale height is smallest in the upper layers, and the density variation therefore greatest.

NEMPI is a large-scale instability that can be excited in stratified small-scale turbulence. This requires (i) sufficient scale separation in the sense that the maximum scale of turbulent motions, $\ell$, must be much smaller than the scale of the system, $L$; and (ii) strong density stratification such that the density scale height $H_{\rho}$ is much smaller than $L$; i.e.,

$\ell \ll H_{\rho} \ll L$.

However, both the size of turbulent motions and the typical size of perturbations due to NEMPI can be related to the density scale height. Furthermore, earlier work of Kemel et al. (2013) using isothermal layers shows that the scale of perturbations due to NEMPI exceeds the typical density scale height. Unlike the isothermal case, in which the scale height is constant, it decreases rapidly with height in a polytropic layer. It is then unclear how such structures could fit into the narrow space left by the stratification and whether the scalings derived for the isothermal case can still be applied locally to polytropic layers.

NEMPI has already been studied previously for polytropic layers in mean-field simulations (MFS; Brandenburg et al. 2010; Käpylä et al. 2012; Jabbari et al. 2013), but no systematic comparison has been made with NEMPI in isothermal or in polytropic layers with different values of the polytropic index. This will be done in the present paper, both in MFS and DNS. Those two complementary types of simulations have proved to be a good tool for understanding the underlying physics of NEMPI. An example are the studies of the effects of rotation on NEMPI (Losada et al. 2012, 2013), where MFS have been able to give quantitatively useful predictions before corresponding DNS were able to confirm the resulting dependence.

\section{Polytropic stratification}

We discuss here the equation for the vertical profile of the fluid density in a polytropic layer. In a Cartesian plane-parallel layer with polytropic stratification, the temperature gradient is constant, so the temperature goes linearly to zero at $z_{\infty}$. The temperature, $T$, is proportional to the square of the sound speed, $c_{\mathrm{s}}^{2}$, and thus also to the density scale height $H_{\rho}(z)$, which is given by $H_{\rho}=c_{\mathrm{s}}^{2} / g$ for an isentropic stratification, where $\boldsymbol{g}$ is the acceleration due to the gravity. For a perfect gas, the density $\rho$ is proportional to $T^{n}$, and the pressure $p$ is proportional to $T^{n+1}$, such that $p / \rho$ is proportional to $T$, where $n$ is the polytropic index. Furthermore, we have $p(z) \propto \rho(z)^{\Gamma}$, where $\Gamma=(n+1) / n$ is another useful coefficient.

For a perfect gas, the specific entropy can be defined (up to an additive constant) as $s=c_{v} \ln \left(p / \rho^{\gamma}\right)$, where $\gamma=c_{p} / c_{v}$ is the ratio of specific heats at constant pressure and constant density, respectively. For a polytropic stratification, we have

$\exp \left(s / c_{v}\right)=p / \rho^{\gamma} \propto \rho^{\Gamma-\gamma}$,

so $s$ is constant when $\Gamma=\gamma$, which is the case for an isentropic stratification. In the following, we make this assumption and specify from now only the value of $\gamma$. For a monatomic gas, we have $\gamma=5 / 3$, which is relevant for the Sun, while for a diatomic molecular gas, we have $\gamma=7 / 5$, which is relevant for air. In those cases, a stratification with $\Gamma=\gamma$ can be motivated by assuming perfect mixing across the layer. The isothermal case with $\gamma=1$ can be motivated by assuming rapid heating/cooling to a constant temperature.

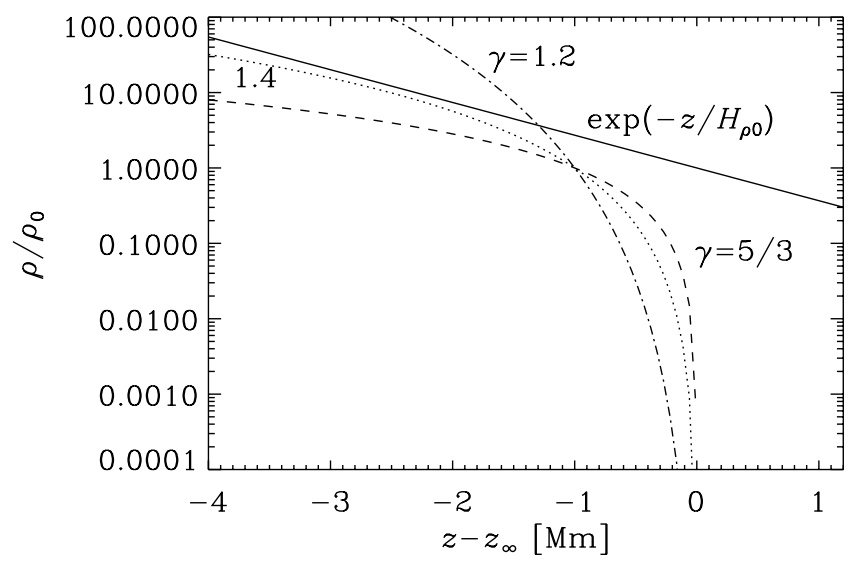

Fig. 1. Isothermal and polytropic relations for different values of $\gamma$ when calculated using the conventional formula $\rho \propto\left(z_{\infty}-z\right)^{n}$ with $n=1 /(\gamma-1)$.

Our aim is to study the change in the properties of NEMPI in a continuous fashion as we go from an isothermally stratified layer to a polytropic one. In the latter case, the fluid density varies in a power law fashion, $\rho \propto\left(z_{\infty}-z\right)^{n}$, while in the former, it varies exponentially, $\rho \propto \exp \left(z_{\infty}-z\right)$. This is shown in Fig. 1 where we compare the exponential isothermal atmosphere with a family of polytropic atmospheres with $\gamma=1.2,1.4$, and 5/3. Clearly, there is no continuous connection between the isothermal case and the polytropic one in the limit $\gamma \rightarrow 1$. This cannot be fixed by rescaling the isothermal density stratification, because in Fig. 1 its values would still lie closer to $5 / 3$ than to 1.4 or 1.2. Another problem with this description is that for polytropic solutions the density is always zero at $z=z_{\infty}$, but finite in the isothermal case. These different behaviors between isothermal and polytropic atmospheres can be unified by using the generalized exponential function known as the " $q$-exponential" (see, e.g., Yamano 2002), which is defined as

$e_{q}(x)=[1+(1-q) x]^{1 /(1-q)}$,

where the parameter $q$ is related to $\gamma$ via $q=2-\gamma$. This generalization of the usual exponential function was originally introduced by Tsallis (1988) in connection with a possible generalization of the Boltzmann-Gibbs statistics. Its usefulness in connection with stellar polytropes has been employed by Plastino \& Plastino (1993). Thus, the density stratification is given by

$\frac{\rho}{\rho_{0}}=\left[1+(\gamma-1)\left(-\frac{z}{H_{\rho 0}}\right)\right]^{1 /(\gamma-1)}=\left(1-\frac{z}{n H_{\rho 0}}\right)^{n}$,

which reduces to $\rho / \rho_{0}=\exp \left(-z / H_{\rho 0}\right)$ for isothermal stratification with $\gamma \rightarrow 1$ and $n \rightarrow \infty$. The density scale height is then given by

$H_{\rho}(z)=H_{\rho 0}-(\gamma-1) z=H_{\rho 0}-z / n$.

In the following, we measure lengths in units of $H_{\rho 0}=H_{\rho}(0)$.

In Fig. 2 we show the dependencies of $\rho(z)$ and $H_{\rho}$ given by Eqs. (4) and (5) for different values of $\gamma$. Compared with Fig. 1, where $z_{\infty}$ is held fixed, in Fig. 2 it is equal to $z_{\infty}=n H_{\rho 0}=$ $H_{\rho 0} /(\gamma-1)$. The total density contrast is roughly the same in all four cases for different $\gamma$, but for increasing values of $\gamma$, the vertical density gradient becomes progressively stronger in the upper layers.

Assuming that the radius $R$ of the resulting structures is proportional to $H_{\rho}$, we sketch in Fig. 3 a situation in which $R$ is 
I. R. Losada et al.: Magnetic flux concentrations in a polytropic atmosphere
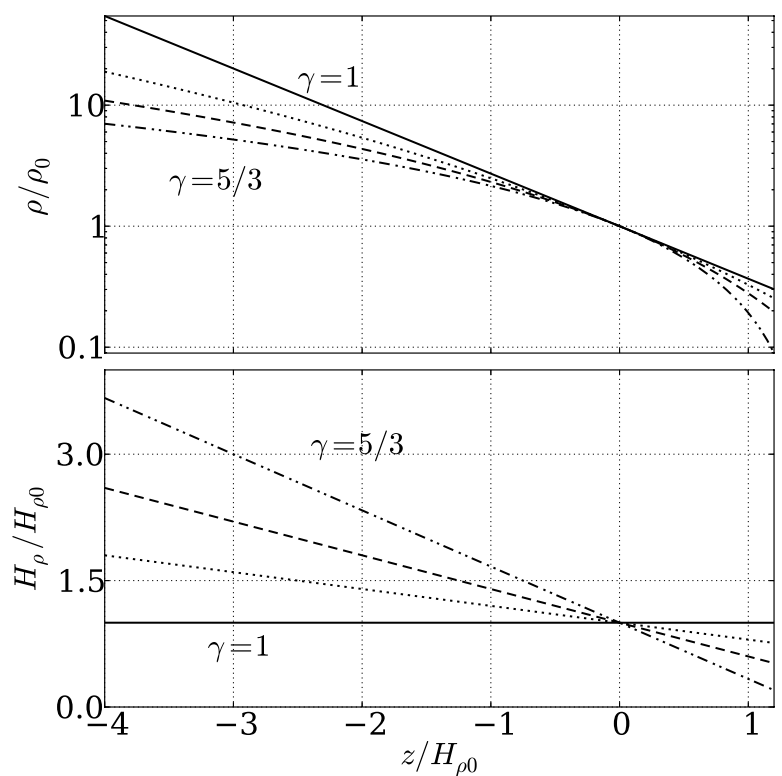

Fig. 2. Polytropes (Eq. (4)) with $\gamma=1$ (solid line), 1.2 (dash-dotted), 1.4 (dotted), and 5/3 (dashed) and density scale height (Eq. (5)) for $-4 \leq$ $-z / H_{\rho 0} \leq 1.2$. The total density contrast is similar for $\gamma=1$ and $5 / 3$.

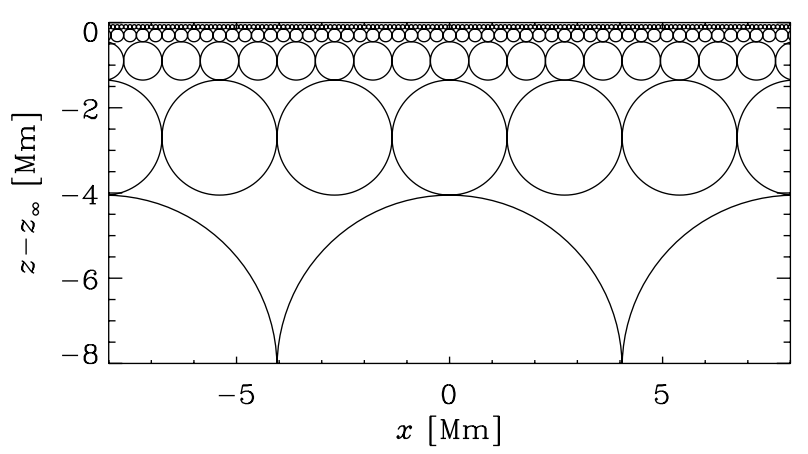

Fig. 3. Sketch showing the expected size distribution of the nearly circular NEMPI eigenfunction structures at different heights.

half the depth. With $\rho \propto\left(z_{\infty}-z\right)^{n}$, the density scale height is $H_{\rho}(z)=\left(z_{\infty}-z\right) / n$. Thus, Fig. 3 applies to a case in which $R=\left(z_{\infty}-z\right) / 2=(n / 2) H_{\rho}(z)$. The solar convection zone is nearly isentropic and well described by $n=3 / 2$. This means that the structures of Fig. 3 have $R=(3 / 4) H_{\rho}(z)$. The results of Kemel et al. (2013) and Brandenburg et al. (2014) suggest that the horizontal wavenumber of structures, $k_{\perp}$, formed by NEMPI, is less than or about $H_{\rho}^{-1}$, so their horizontal wavelength is $\lesssim 2 \pi H_{\rho}$. One wavelength corresponds to the distance between two nodes, i.e., the distance between two spheres, which is $4 R$. Thus, in the isothermal case we have $R / H_{\rho}=2 \pi / 4 \approx 1.5$, which implies that such a structure would not fit into the isentropic atmosphere described above. This provides an additional motivation for our present work.

\section{DNS study}

In this section we study NEMPI in DNS for the polytropic layer. Corresponding MFS are presented in Sect. 4.

\subsection{The model}

We solve the equations for the magnetic vector potential $\boldsymbol{A}$, the velocity $\boldsymbol{U}$, and the density $\rho$, in the form

$$
\begin{aligned}
& \frac{\partial \boldsymbol{A}}{\partial t}=\boldsymbol{U} \times \boldsymbol{B}-\eta \mu_{0} \boldsymbol{J}, \\
& \frac{\mathrm{D} \boldsymbol{U}}{\mathrm{D} t}=\frac{1}{\rho} \boldsymbol{J} \times \boldsymbol{B}+\boldsymbol{f}-v \boldsymbol{Q}-\boldsymbol{\nabla} H, \\
& \frac{\mathrm{D} \rho}{\mathrm{D} t}=-\rho \boldsymbol{\nabla} \cdot \boldsymbol{U},
\end{aligned}
$$

where $\mathrm{D} / \mathrm{D} t=\partial / \partial t+\boldsymbol{U} \cdot \boldsymbol{\nabla}$ is the advective derivative with respect to the actual (turbulent) flow, $\boldsymbol{B}=\boldsymbol{B}_{0}+\boldsymbol{\nabla} \times \boldsymbol{A}$ is the magnetic field, $\boldsymbol{B}_{0}$ the imposed uniform field, $\boldsymbol{J}=\boldsymbol{\nabla} \times \boldsymbol{B} / \mu_{0}$ the current density, $\mu_{0}$ the vacuum permeability, $H=h+\Phi$ the reduced enthalpy, $h=c_{p} T$ the enthalpy, $\Phi$ is the gravitational potential,

$-\boldsymbol{Q}=\nabla^{2} \boldsymbol{U}+\frac{1}{3} \boldsymbol{\nabla} \boldsymbol{\nabla} \cdot \boldsymbol{U}+2 \boldsymbol{S} \boldsymbol{\nabla} \ln \rho$

is a term appearing in the viscous force $-v \boldsymbol{Q}, \mathbf{S}$ is the traceless rate-of-strain tensor with components

$\mathrm{S}_{i j}=\frac{1}{2}\left(\nabla_{j} U_{i}+\nabla_{i} U_{j}\right)-\frac{1}{3} \delta_{i j} \boldsymbol{\nabla} \cdot \boldsymbol{U}$,

$v$ is the kinematic viscosity, and $\eta$ is the magnetic diffusion coefficient caused by electrical conductivity of the fluid. As in Losada et al. (2012), $z$ corresponds to radius, $x$ to colatitude, and $y$ to azimuth. The forcing function $f$ consists of random, white-in-time, plane, nonpolarized waves with a certain average wavenumber $k_{\mathrm{f}}$.

\subsection{Boundary conditions and parameters}

In the DNS we use stress-free boundary conditions for the velocity at the top and bottom; i.e., $\nabla_{z} U_{x}=\nabla_{z} U_{y}=U_{z}=0$. For the magnetic field we use either perfect conductor boundary conditions, $A_{x}=A_{y}=\nabla_{z} A_{z}=0$, or vertical field conditions, $\nabla_{z} A_{x}=\nabla_{z} A_{y}=A_{z}=0$, again at both the top and bottom. All variables are assumed periodic in the $x$ and $y$ directions.

The turbulent rms velocity is approximately independent of $z$ with $u_{\mathrm{rms}}=\left\langle\boldsymbol{u}^{2}\right\rangle^{1 / 2} \approx 0.1 c_{\mathrm{s}}$. The gravitational acceleration $\boldsymbol{g}=(0,0,-g)$ is chosen such that $k_{1} H_{\rho 0}=1$, where $k_{1}=2 \pi / L$ and $L$ is the size of the domain. With one exception (Sect. 3.5), we always use the value $k_{\mathrm{f}} / k_{1}=30$ for the scale separation ratio. For $\boldsymbol{B}_{0}$ we choose either a horizontal field pointing in the $y$ direction or a vertical one pointing in the $z$ direction. The latter case, $\boldsymbol{B}_{0}=\left(0,0, B_{0}\right)$, is usually combined with the use of the vertical field boundary condition, while the former one, $\boldsymbol{B}_{0}=\left(0, B_{0}, 0\right)$, is combined with using perfect conductor boundary conditions. The strength of the imposed field is expressed in terms of $B_{\text {eq } 0}=$ $B_{\text {eq }}(z=0)$, which is the equipartition field strength at $z=0$. Here, the equipartition field $B_{\text {eq }}(z)=\left(\mu_{0} \bar{\rho}(z)\right)^{1 / 2} u_{\text {rms }}$. The imposed field is normalized by $B_{\text {eq0 }}$ and denoted as $\beta_{0}=B_{0} / B_{\text {eq } 0}$, while $\beta=|\overline{\boldsymbol{B}}| / B_{\text {eq }}$ is the modulus of the normalized mean magnetic field. Time is expressed in terms of the turbulent-diffusive time, $\tau_{\mathrm{td}}=H_{\rho 0}^{2} / \eta_{\mathrm{t} 0}$, where $\eta_{\mathrm{t} 0}=u_{\mathrm{rms}} / 3 k_{\mathrm{f}}$ (Sur et al. 2008) is an estimate for the turbulent magnetic diffusivity used in the DNS.

Our values of $v$ and $\eta$ are characterized by specifying the kinetic and magnetic Reynolds numbers,

$\operatorname{Re}=u_{\mathrm{rms}} / \nu k_{\mathrm{f}}, \quad R_{\mathrm{m}}=u_{\mathrm{rms}} / \eta k_{\mathrm{f}}$.

In most of this paper (except in Sect. 3.5) we use $\mathrm{Re}=36$ and $R_{\mathrm{m}}=18$, which are also the values used by Kemel et al. (2013). 

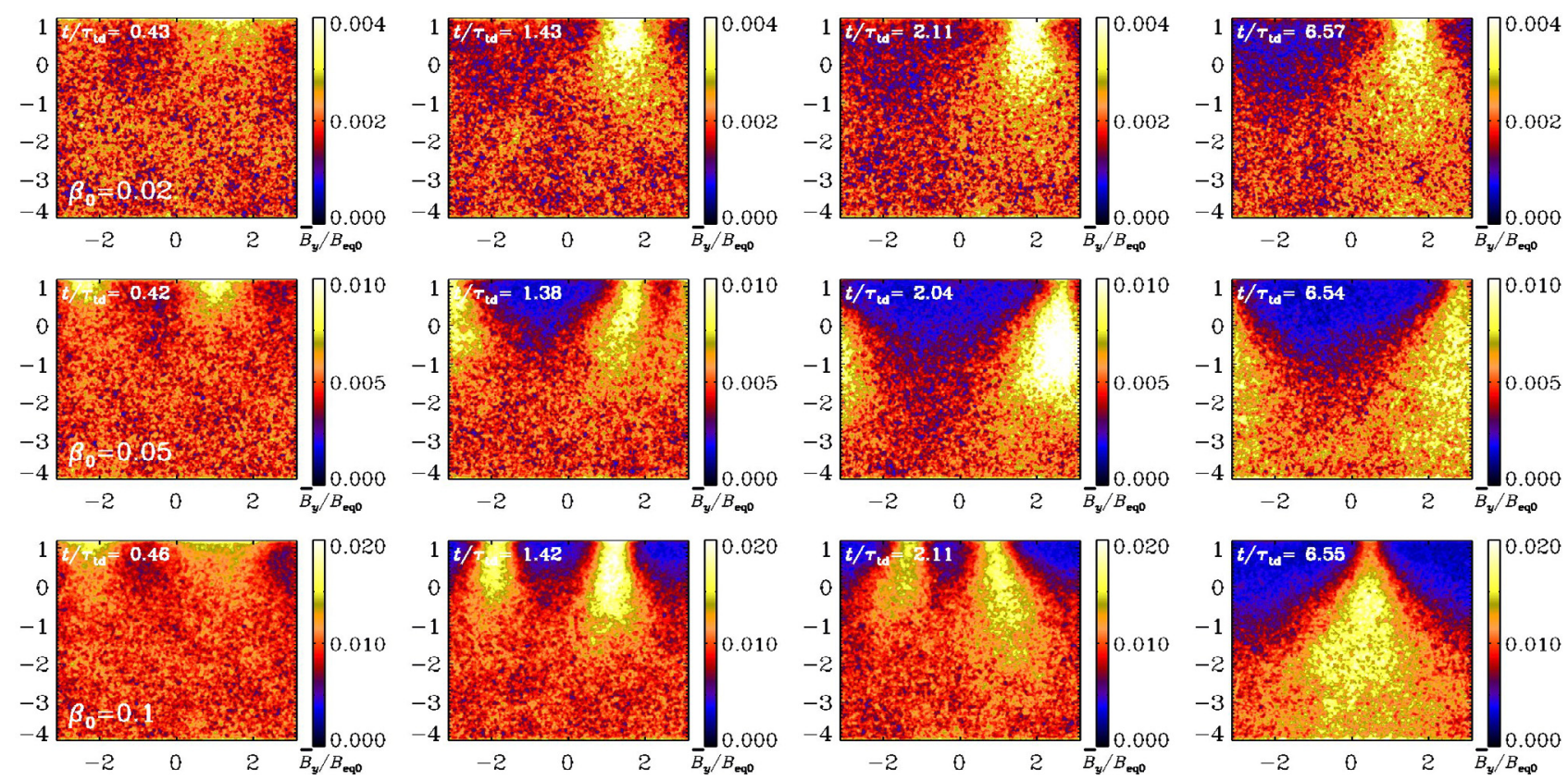

Fig. 4. Snapshots of $\bar{B}_{y}$ from DNS for $\gamma=5 / 3$ and $\beta_{0}=0.02$ (upper row), 0.05 (middle row), and 0.1 (lower row) at different times (indicated in turbulent-diffusive times, increasing from left to right) in the presence of a horizontal field using the perfect conductor boundary condition.

The DNS are performed with the PENCIL CODE, http:// pencil-code.googlecode.com, which uses sixth-order explicit finite differences in space and a third-order accurate time stepping method. We use a numerical resolution of $256^{3}$ mesh points.

\subsection{Horizontal fields}

We focus on the case $\gamma=5 / 3$ and show in Fig. 4 visualizations of $\bar{B}_{y}$ at different instants for three values of the imposed horizontal magnetic field strength. For $\beta_{0}=0.02$, a magnetic structure is clearly visible at $t / \tau_{\mathrm{td}}=1.43$, while for $\beta_{0}=0.05$ structures are already fully developed at $t / \tau_{\mathrm{td}}=0.42$. In that case $\left(\beta_{0}=0.05\right)$, at early times $\left(t / \tau_{\mathrm{td}}=0.42\right)$, there are two structures, which then begin to merge at $t / \tau_{\mathrm{td}}=1.38$. The growth rate of the magnetic structure is found to be $\lambda \approx 2 \eta_{\mathrm{t} 0} / H_{\rho 0}^{2}$ for the runs shown in Fig. 4. This is less than the value of $\lambda \approx 5 \eta_{t 0} / H_{\rho 0}^{2}$ found earlier for the isothermal case (Kemel et al. 2012b).

For $\gamma=5 / 3$ and $\beta_{0} \leq 0.02$, the magnetic structures become smaller $\left(k_{\perp} H_{\rho 0}=2\right)$ near the surface. In the nonlinear regime, i.e., at late times, the structures move downward due to the so-called "potato-sack" effect, which was first seen in MFS (Brandenburg et al. 2010) and later confirmed in DNS (Brandenburg et al. 2011). The magnetic structures sink in the nonlinear stage of NEMPI, because an increase in the mean magnetic field inside the horizontal magnetic flux tube increases the absolute value of the effective magnetic pressure. On the other hand, a decrease in the negative effective magnetic pressure is balanced out by increased gas pressure, which in turn leads to higher density, so the magnetic structures become heavier than the surroundings and sink. This potato-sack effect has been clearly observed in the present DNS with the polytropic layer (see the right column in Fig. 4).

\subsection{Vertical fields}

Recent DNS using isothermal layers have shown that strong circular flux concentrations can be produced in the case of a vertical magnetic field (Brandenburg et al. 2013, 2014). This is also observed in the present study of a polytropic layer; see Fig. 5, where we show the evolution of $\bar{B}_{z}$ on the periphery of the computational domain for $\gamma=5 / 3$ and $\beta_{0}=0.05$ at different times. A difference to the DNS for $\gamma=1$ (Brandenburg et al. 2013) seems to be that for $\gamma=5 / 3$ the magnetic structures are shallower than for $\gamma=1$; see Fig. 6, where we show $x y$ and $x z$ slices of $B_{z}$ through the spot. Owing to periodicity in the $x y$ plane, we have shifted the location of the spot to $x=y=0$. We note also that the field lines of the averaged magnetic field show a structure rather similar to the one found in MFS of Brandenburg et al. (2014). The origin of circular structures is associated with a cylindrical symmetry for the vertical magnetic field. The growth rate of the magnetic field in the spot is found to be $\lambda \approx 0.9 \eta_{\mathrm{t} 0} / H_{\rho 0}^{2}$, which is similar to the value of 1.3 found earlier for the isothermal case (Brandenburg et al. 2013).

\subsection{Effective magnetic pressure}

As pointed out in Sect. 1, the main reason for the formation of strongly inhomogeneous large-scale magnetic structures is the negative contribution of turbulence to the large-scale magnetic pressure, so that the effective magnetic pressure (the sum of turbulent and nonturbulent contributions) can be negative at high magnetic Reynolds numbers. The effective magnetic pressure has been determined from DNS for isothermally stratified forced turbulence (Brandenburg et al. 2010, 2012) and for turbulent convection (Käpylä et al. 2012). To see whether the nature of polytropic stratification has any influence on the effective magnetic pressure, we use DNS.

We first explain the essence of the effect of turbulence on the effective magnetic pressure. We consider the momentum equation in the form

$\frac{\partial}{\partial t} \rho U_{i}=-\frac{\partial}{\partial x_{j}} \Pi_{i j}+\rho g_{i}$

where

$\Pi_{i j}=\rho U_{i} U_{j}+\delta_{i j}\left(p+\boldsymbol{B}^{2} / 2 \mu_{0}\right)-B_{i} B_{j} / \mu_{0}-2 v \rho \mathrm{S}_{i j}$

is the momentum stress tensor and $\delta_{i j}$ the Kronecker tensor. 
I. R. Losada et al.: Magnetic flux concentrations in a polytropic atmosphere
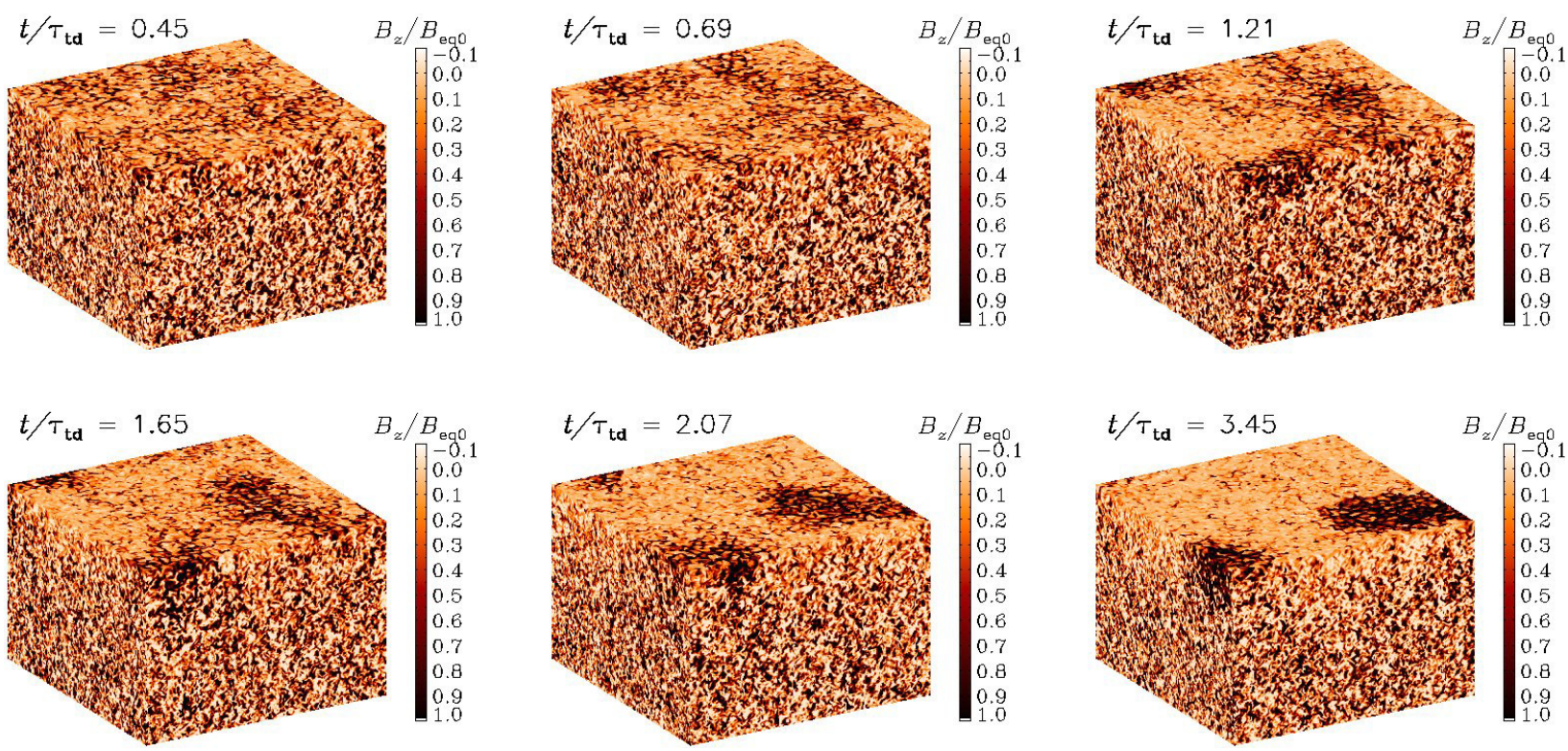

Fig. 5. Snapshots from DNS showing $\bar{B}_{z}$ on the periphery of the computational domain for $\gamma=5 / 3$ and $\beta_{0}=0.05$ at different times for the case of a vertical field using the vertical field boundary condition.
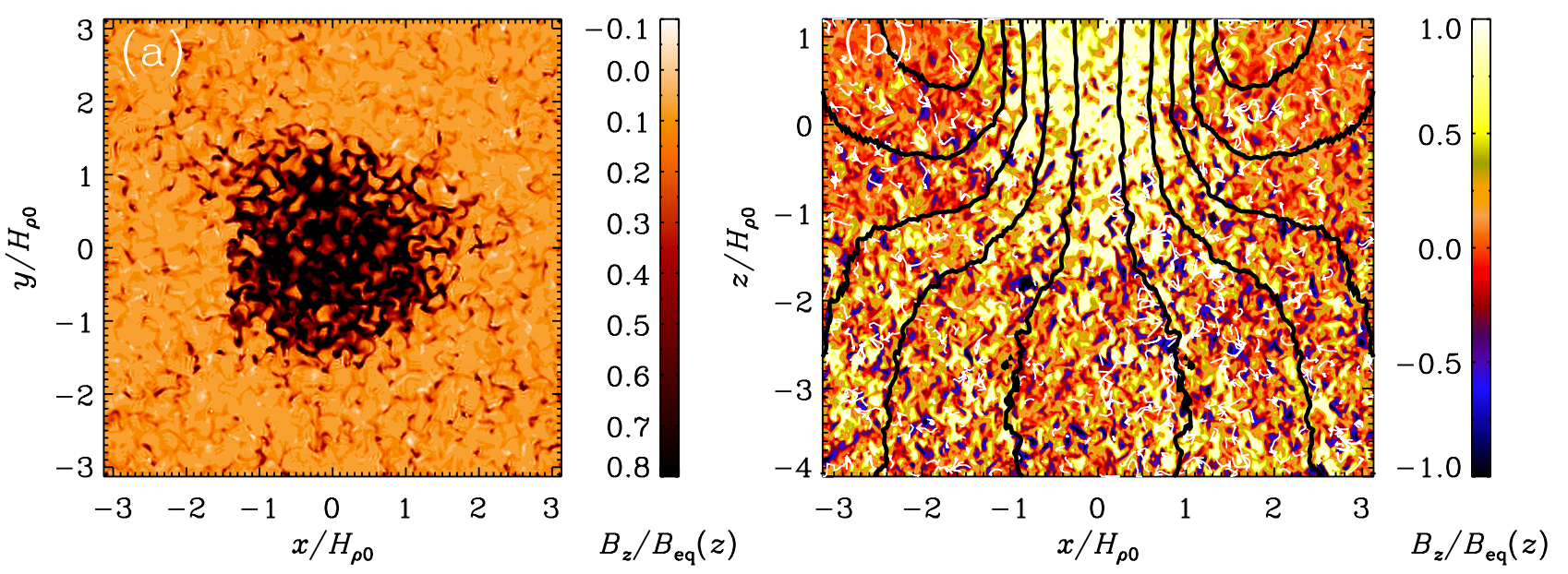

Fig. 6. Cuts of $B_{z} / B_{\text {eq }}(z)$ in (a) the $x y$ plane at the top boundary $\left(z / H_{\rho 0}=1.2\right)$ and (b) the $x z$ plane through the middle of the spot at $y=0$ for $\gamma=5 / 3$ and $\beta_{0}=0.05$. In the $x z$ cut, we also show magnetic field lines and flow vectors obtained by numerically averaging in azimuth around the spot axis.

Ignoring correlations between velocity and density fluctuations for low-Mach number turbulence, the averaged momentum equation is

$\frac{\partial}{\partial t} \bar{\rho} \overline{\boldsymbol{U}}_{i}=-\frac{\partial}{\partial x_{j}} \bar{\Pi}_{i j}+\bar{\rho} g_{i}$

where an overbar means $x y$ averaging, $\bar{\Pi}_{i j}=\bar{\Pi}_{i j}^{\mathrm{m}}+\bar{\Pi}_{i j}^{\mathrm{f}}$ is the mean momentum stress tensor, split into contributions resulting from the mean field (indicated by superscript $\mathrm{m}$ ) and the fluctuating field (indicated by superscript f). The tensor $\bar{\Pi}_{i j}^{\mathrm{m}}$ has the same form as Eq. (13), but all quantities have now attained an overbar; i.e.,

$\bar{\Pi}_{i j}^{\mathrm{m}}=\bar{\rho} \bar{U}_{i} \bar{U}_{j}+\delta_{i j}\left(\bar{p}+\overline{\boldsymbol{B}}^{2} / 2 \mu_{0}\right)-\bar{B}_{i} \bar{B}_{j} / \mu_{0}-2 v \bar{\rho} \overline{\mathrm{S}}_{i j}$

The contributions, $\bar{\Pi}_{i j}^{\mathrm{f}}$, which result from the fluctuations $\boldsymbol{u}=$ $\boldsymbol{U}-\overline{\boldsymbol{U}}$ and $\boldsymbol{b}=\boldsymbol{B}-\overline{\boldsymbol{B}}$ of velocity and magnetic fields, respectively, are determined by the sum of the Reynolds and Maxwell stress tensors:

$\bar{\Pi}_{i j}^{\mathrm{f}}=\bar{\rho} \overline{u_{i} u_{j}}+\delta_{i j} \overline{\boldsymbol{b}^{2}} / 2 \mu_{0}-\overline{b_{i} b_{j}} / \mu_{0}$.

This contribution, together with the contribution from the mean field, $\bar{\Pi}_{i j}^{\mathrm{m}}$, comprises the total mean momentum tensor. The contribution from the fluctuating fields is split into a contribution that is independent of the mean magnetic field $\bar{\Pi}_{i j}^{\mathrm{f}, 0}$ (which determines the turbulent viscosity and background turbulent pressure) and a contribution that depends on the mean magnetic field $\bar{\Pi}_{i j}^{\mathrm{f}, \bar{B}}$. The difference between the two, $\Delta \bar{\Pi}_{i j}^{\mathrm{f}}=\bar{\Pi}_{i j}^{\mathrm{f}, \bar{B}}-\bar{\Pi}_{i j}^{\mathrm{f}, 0}$, is caused by the mean magnetic field and is parameterized in the form

$\Delta \bar{\Pi}_{i j}^{\mathrm{f}}=\mu_{0}^{-1}\left(q_{\mathrm{s}} \bar{B}_{i} \bar{B}_{j}-q_{\mathrm{p}} \delta_{i j} \overline{\boldsymbol{B}}^{2} / 2-q_{\mathrm{g}} \hat{g}_{i} \hat{g}_{j} \overline{\boldsymbol{B}}^{2}\right)$,

where the coefficient $q_{\mathrm{p}}$ represents the isotropic turbulence contribution to the mean magnetic pressure, the coefficient $q_{\mathrm{s}}$ represents the turbulence contribution to the mean magnetic tension, 


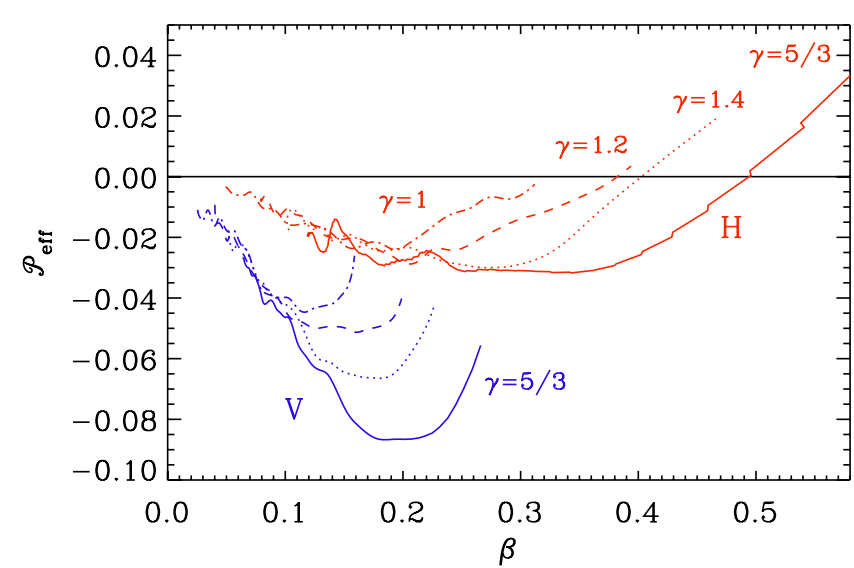

Fig. 7. Effective magnetic pressure obtained from DNS in a polytropic layer with different $\gamma$ for horizontal ( $\mathrm{H}$, red curves) and vertical (V, blue curves) mean magnetic fields.

while the coefficient $q_{\mathrm{g}}$ is the anisotropic turbulence contribution to the mean magnetic pressure, and it characterizes the effect of vertical variations of the magnetic field caused by the vertical magnetic pressure gradient. Here, $\hat{g}_{i}$ is the unit vector in the direction of the gravity field (in the vertical direction). We consider cases with horizontal and vertical mean magnetic fields separately. Analytically, the coefficients $q_{\mathrm{p}}, q_{\mathrm{g}}$, and $q_{\mathrm{s}}$ have been obtained using both the spectral $\tau$ approach (Rogachevskii $\&$ Kleeorin 2007) and the renormalization approach (Kleeorin \& Rogachevskii 1994). The form of Eq. (17) is also obtained using simple symmetry arguments; e.g., for a horizontal field, the linear combination of three independent true tensors, $\delta_{i j}, \hat{g}_{i} \hat{g}_{j}$ and $\bar{B}_{i} \bar{B}_{j}$, yields Eq. (17), while for the vertical field, the linear combination of only two independent true tensors, $\delta_{i j}$ and $\bar{B}_{i} \bar{B}_{j}$.

Previous DNS studies (Brandenburg et al. 2012) have shown that $q_{\mathrm{s}}$ and $q_{\mathrm{g}}$ are negligible for forced turbulence. To avoid the formation of magnetic structures in the nonlinear stage of NEMPI, which would modify our results, we use here a lower scale separation ratio, $k_{\mathrm{f}} / k_{1}=5$, keeping $k_{1} H_{\rho}=1$, and using $\operatorname{Re}=140$ and $R_{\mathrm{m}}=70$, as in Brandenburg et al. (2012). To determine $q_{\mathrm{p}}(\beta)$, it is sufficient to measure the three diagonal components of $\bar{\Pi}_{i j}^{\mathrm{f}}$ both with and without an imposed magnetic field using $q_{\mathrm{p}}=-2 \mu_{0} \Delta \bar{\Pi}_{x x}^{\mathrm{f}} / \overline{\boldsymbol{B}}^{2}$.

In Fig. 7 we present the results for forced turbulence in the polytropic layer with different $\gamma$ for horizontal and vertical mean magnetic fields. It turns out that the normalized effective magnetic pressure,

$\mathcal{P}_{\text {eff }}=\frac{1}{2}\left(1-q_{\mathrm{p}}\right) \beta^{2}$,

has a minimum value $\mathcal{P}_{\min }$ at $\beta_{\text {min }}$. Following Kemel et al. (2012a), the function $q_{\mathrm{p}}(\beta)$ is approximated by:

$q_{\mathrm{p}}(\beta)=\frac{q_{\mathrm{p} 0}}{1+\beta^{2} / \beta_{\mathrm{p}}^{2}}=\frac{\beta_{\star}^{2}}{\beta_{\mathrm{p}}^{2}+\beta^{2}}$,

where $q_{\mathrm{p} 0}, \beta_{\mathrm{p}}$, and $\beta_{\star}=\beta_{\mathrm{p}} q_{\mathrm{p} 0}^{1 / 2}$ are constants. This equation can be understood as a quenching formula for the effective magnetic pressure; see Jabbari et al. (2013). The coefficients $\beta_{\mathrm{p}}$ and $\beta_{\star}$ are related to $\mathcal{P}_{\min }$ at $\beta_{\min }$ via (Kemel et al. 2012a)

$\beta_{\mathrm{p}}=\beta_{\min }^{2} / \sqrt{-2 \mathcal{P}_{\min }}, \quad \beta_{\star}=\beta_{\mathrm{p}}+\sqrt{-2 \mathcal{P}_{\text {min }}}$.
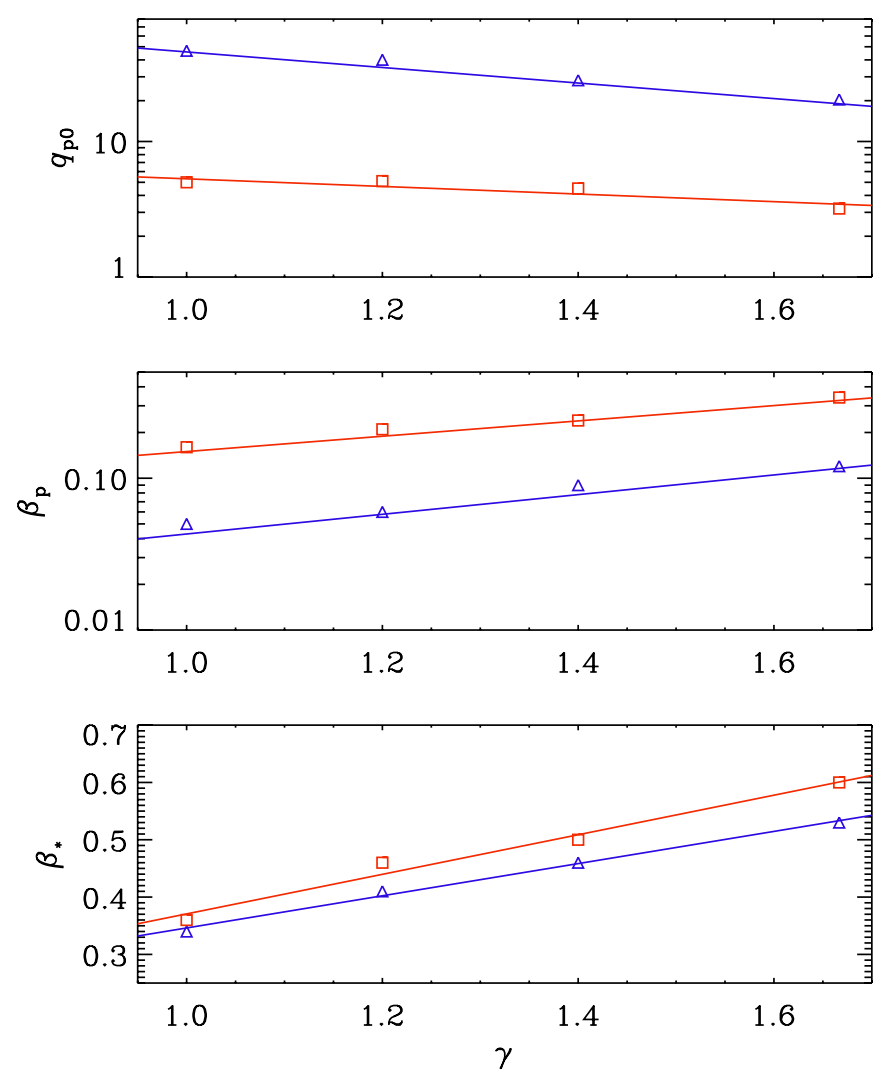

Fig. 8. Parameters $q_{\mathrm{p} 0}, \beta_{\mathrm{p}}$, and $\beta_{\star}$ for the function $q_{\mathrm{p}}(\beta)$ (see Eq. (19)) versus $\gamma$ for horizontal (red line) and vertical (blue line) mean magnetic fields.

In Fig. 8 we show these fitting parameters for the function $q_{\mathrm{p}}(\beta)$ for polytropic layer with different $\gamma$ for horizontal and vertical mean magnetic fields. The effects of negative effective magnetic pressure are generally stronger for vertical magnetic fields $\left(q_{\mathrm{p} 0}\right.$ is larger and $\beta_{\mathrm{p}}$ smaller) than for horizontal ones ( $q_{\mathrm{p} 0}$ is smaller and $\beta_{\mathrm{p}}$ larger), but the values $\beta_{\star}=\beta_{\mathrm{p}} q_{\mathrm{p} 0}^{1 / 2}$ are similar in both cases, and increasing from 0.35 (for $\gamma=1$ ) to 0.6 (for $\gamma=5 / 3$ ); see Fig. 8.

\section{Mean-field study}

We now consider two sets of parameters that we refer to as Model I (with $q_{\mathrm{p} 0}=32$ and $\beta_{\mathrm{p}}=0.058$ corresponding to $\left.\beta_{\star}=0.33\right)$ and Model II $\left(q_{\mathrm{p} 0}=9\right.$ and $\beta_{\mathrm{p}}=0.21$ corresponding to $\left.\beta_{\star}=0.63\right)$. These cases are representative of the strong (large $\beta_{\star}$ ) and weak (small $\beta_{\star}$ ) effects of NEMPI. Following earlier studies (Brandenburg et al. 2012), we find $q_{\mathrm{s}}$ to be compatible with zero. We thus neglect this coefficient in the following.

\subsection{Governing parameters and estimates}

The purpose of this section is to summarize the findings for the isothermal case in MFS. One of the key results is the prediction of the growth rate of NEMPI. The work of Kemel et al. (2013) showed that in the ideal case (no turbulent diffusion), the growth rate $\lambda$ is approximated well by

$\lambda \approx \beta_{\star} u_{\mathrm{rms}} / H_{\rho} \quad$ (no turbulent diffusion) 
However, turbulent magnetic diffusion, $\eta_{\mathrm{t}}$, can clearly not be neglected and is chiefly responsible for shutting off NEMPI if the turbulent eddies are too big and $\eta_{\mathrm{t}}$ too large. This was demonstrated in Fig. 17 of Brandenburg et al. (2012). A heuristic ansatz, which is motivated by similar circumstances in meanfield dynamo theory (Krause \& Rädler 1980), is to add a term $-\eta_{\mathrm{t}} k^{2}$ to the righthand side of Eq. (21), where $k$ is the wavenumber of NEMPI.

To specify the expression for $\lambda$, we normalize the wavenumber of the perturbations by the inverse density scale height and denote this by $\kappa \equiv k H_{\rho}$. The wavenumber of the energy-carrying turbulent eddies $k_{\mathrm{f}}$ is in nondimensional form $\kappa_{\mathrm{f}} \equiv k_{\mathrm{f}} H_{\rho}$, and the normalized horizontal wavenumber of the resulting magnetic structures is referred to as $\kappa_{\perp}=k_{\perp} H_{\rho}$. For NEMPI, these values have been estimated to be $\kappa_{\perp}=0.8-1.0$, and can even be smaller for vertical magnetic fields (Brandenburg et al. 2014). Using an approximate aspect ratio of unity for magnetic structures, we have $\kappa=\sqrt{2} \kappa_{\perp} \approx 1.1-1.4$. In stellar mixing length theory (Vitense 1953), the mixing length is $\ell_{\mathrm{f}}=\alpha_{\text {mix }} H_{\rho}$, where $\alpha_{\text {mix }} \approx 1.6$ is a nondimensional mixing length parameter. Since $k_{\mathrm{f}}=2 \pi / \ell_{\mathrm{f}}$, we arrive at the following estimate: $\kappa_{\mathrm{f}}=2 \pi \gamma / \alpha_{\text {mix }} \approx 6.5$. [Owing to a confusion between pressure and density scale heights, this value was underestimated by Kemel et al. (2013) to be 2.4, although an independent calculation of this value from turbulent convection simulations would still be useful.] Using $\eta_{\mathrm{t}} \approx u_{\mathrm{rms}} / 3 k_{\mathrm{f}}$, the turbulent magnetic diffusive rate for an isothermal atmosphere is given by

$\eta_{\mathrm{t}} k^{2}=\frac{u_{\mathrm{rms}}}{3 H_{\rho}} \frac{\kappa^{2}}{\kappa_{\mathrm{f}}}$,

and the growth rate of NEMPI in that normalization is

$\frac{\lambda}{\eta_{\mathrm{t}} k^{2}}=3 \beta_{\star} \frac{\kappa_{\mathrm{f}}}{\kappa^{2}}-1$

Using $\beta_{\star}=0.23$, which is the relevant value for high magnetic Reynolds numbers (Brandenburg et al. 2012), we find $\lambda / \eta_{\mathrm{t}} k^{2} \approx 2.7-1.3$ for $\kappa \approx 1.1-1.4$. However, since Fig. 8 shows an increase of $\beta_{\star}$ with increasing polytropic index, one might expect a corresponding increase in the growth rate of NEMPI for a polytropic layer, in which $H_{\rho}$ varies strongly with height $z$. Indeed, in a polytropic atmosphere, $H_{\rho}$ is proportional to depth. Thus, at any given depth there is a layer beneath, where the stratification is less strong and the growth rate of NEMPI is lower. In addition, there is a thinner, more strongly stratified layer above, where NEMPI might grow faster if only the structures generated by NEMPI have enough room to develop before they touch the top of the atmosphere at $z_{\infty}$, where the temperature vanishes.

\subsection{Mean-field equations}

In the following, we consider MFS and compare it with DNS. We also compare our MFS results with those of DNS (Brandenburg et al. 2011; Kemel et al. 2013) using a similar polytropic setup.

The governing equations for the mean quantities (denoted by an overbar) are fairly similar to those for the original equations, except that in the MFS viscosity and magnetic diffusivity are replaced by their turbulent counterparts, and the mean Lorentz force is supplemented by a parameterization of the turbulent contribution to the effective magnetic pressure.
The evolution equations for mean vector potential $\overline{\boldsymbol{A}}$, mean velocity $\overline{\boldsymbol{U}}$, and mean density $\bar{\rho}$, are

$$
\begin{aligned}
& \frac{\partial \overline{\boldsymbol{A}}}{\partial t}=\overline{\boldsymbol{U}} \times \overline{\boldsymbol{B}}-\eta_{\mathrm{T}} \mu_{0} \overline{\boldsymbol{J}}, \\
& \frac{\overline{\mathrm{D}} \overline{\boldsymbol{U}}}{\overline{\mathrm{D}} t}=\frac{1}{\bar{\rho}}\left[\overline{\boldsymbol{J}} \times \overline{\boldsymbol{B}}+\boldsymbol{\nabla}\left(q_{\mathrm{p}} \overline{\boldsymbol{B}}^{2} / 2 \mu_{0}\right)\right]-\nu_{\mathrm{T}} \overline{\boldsymbol{Q}}-\boldsymbol{\nabla} \overline{\boldsymbol{H}}, \\
& \frac{\overline{\mathrm{D}} \bar{\rho}}{\overline{\mathrm{D}} t}=-\bar{\rho} \boldsymbol{\nabla} \cdot \overline{\boldsymbol{U}},
\end{aligned}
$$

where $\overline{\mathrm{D}} / \overline{\mathrm{D}} t=\partial / \partial t+\overline{\boldsymbol{U}} \cdot \boldsymbol{\nabla}$ is the advective derivative with respect to the mean flow, $\bar{\rho}$ the mean density, $\bar{H}=\bar{h}+\Phi$ the mean reduced enthalpy, $\bar{h}=c_{p} \bar{T}$ the mean enthalpy, $\bar{T} \propto \bar{\rho}^{\gamma-1}$ the mean temperature, $\Phi$ the gravitational potential, $\eta_{\mathrm{T}}=\eta_{\mathrm{t}}+\eta$, and $v_{\mathrm{T}}=v_{\mathrm{t}}+v$ are the sums of turbulent and microphysical values of magnetic diffusivity and kinematic viscosities, respectively. Also, $\overline{\boldsymbol{J}}=\boldsymbol{\nabla} \times \overline{\boldsymbol{B}} / \mu_{0}$ is the mean current density, $\mu_{0}$ is the vacuum permeability,

$$
-\overline{\boldsymbol{Q}}=\nabla^{2} \overline{\boldsymbol{U}}+\frac{1}{3} \boldsymbol{\nabla} \boldsymbol{\nabla} \cdot \overline{\boldsymbol{U}}+2 \overline{\mathbf{S}} \boldsymbol{\nabla} \ln \bar{\rho}
$$

is a term appearing in the mean viscous force $-\nu_{\mathrm{T}} \overline{\boldsymbol{Q}}$, where $\overline{\mathbf{S}}$ is the traceless rate-of-strain tensor of the mean flow with components $\overline{\mathrm{S}}_{i j}=\frac{1}{2}\left(\nabla_{j} \bar{U}_{i}+\nabla_{i} \bar{U}_{j}\right)-\frac{1}{3} \delta_{i j} \boldsymbol{\nabla} \cdot \overline{\boldsymbol{U}}$, and finally the term $\boldsymbol{\nabla}\left(q_{\mathrm{p}} \overline{\boldsymbol{B}}^{2} / 2 \mu_{0}\right)$ on the righthand side of Eq. (25) determines the turbulent contribution to the effective magnetic pressure. Here, $q_{\mathrm{p}}$ depends on the local field strength; see Eq. (19). This term enters with a plus sign, so positive values of $q_{\mathrm{p}}$ correspond to a suppression of the total turbulent pressure. The net effect of the mean magnetic field leads to an effective mean magnetic pressure that becomes negative for $q_{\mathrm{p}}>1$. This can indeed be the case for magnetic Reynolds numbers well above unity (Brandenburg et al. 2012); see also Fig. 7 for a polytropic atmosphere.

The boundary conditions for MFS are the same as for DNS, i.e., stress-free for the mean velocity at the top and bottom. For the mean magnetic field, we use either perfect conductor boundary conditions (for horizontal, imposed magnetic fields) or vertical field conditions (for vertical, imposed fields) at the top and bottom. All mean-field variables are assumed to be periodic in the $x$ and $y$ directions. The MFS are performed again with the PENCIL CODE, which is equipped with a mean-field module for solving the corresponding equations.

\subsection{Expected vertical dependence of NEMPI}

To get an idea about the vertical dependence of NEMPI, we now consider the resulting dependencies of $\mathcal{P}_{\text {eff }}(z)$; see the lefthand panels of Fig. 9. We note that $\mathcal{P}_{\text {eff }}$ is just a function of $\beta$ (Eqs. (18) and (19)), which allows us to approximate the local growth rates as (Rogachevskii \& Kleeorin 2007; Kemel et al. 2013)

$\lambda_{0}=\frac{v_{\mathrm{A}}}{H_{\rho 0}}\left(-2 \frac{\mathrm{d} \mathcal{P}_{\mathrm{eff}}}{\mathrm{d} \beta^{2}}\right)^{\frac{1}{2}}$,

which are plotted in the righthand panels of Fig. 9 for Model I.

\subsection{Horizontal fields}

To analyze the kinematic stage of MFS, we measure the value of the maximum downflow speed, $|\bar{U}|_{\max }^{\text {down }}$ at each height. We then 

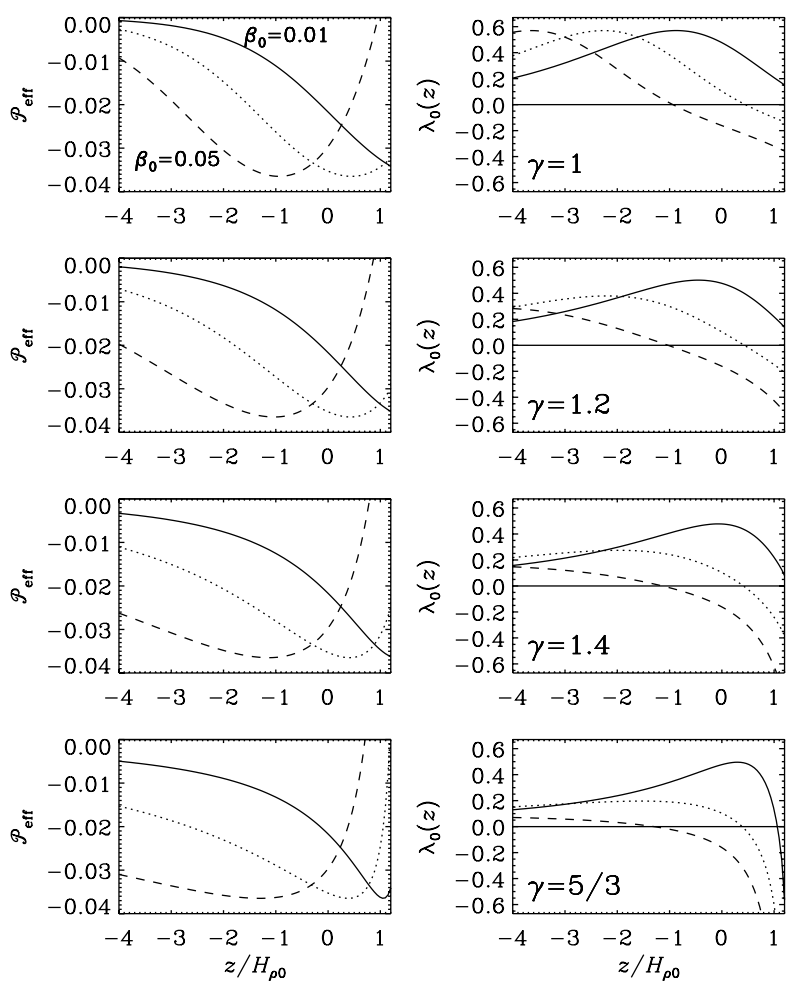

Fig. 9. Comparison of $\mathcal{P}_{\text {eff }}(z)$ and $\lambda_{0}(z)$ in MFS for polytropic layers with four values of $\gamma$ (top to bottom) and three values of $\beta$ (different line types).
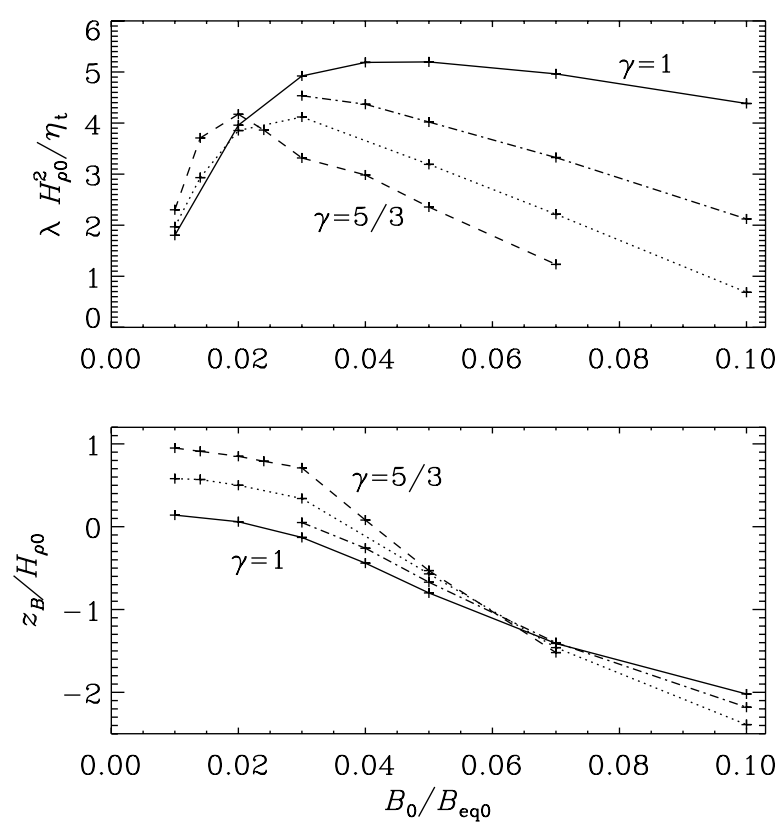

Fig. 10. Dependence of growth rate and height where the eigenfunction attains its maximum value (the optimal depth of NEMPI) on field strength from MFS for different values of $\gamma$ in the presence of a horizontal field for Model I.

determine the time interval during which the maximum downflow speed increases exponentially and when the height of the peak is constant and equal to $z_{B}$. This yields the growth rate of the instability as $\lambda=\mathrm{d} \ln |\bar{U}|_{\max }^{\text {down }} / \mathrm{d} t$.

In Figs. 10 and 11 we plot, respectively for Models I and II, $\lambda$ (in units of $\eta_{\mathrm{t}} / H_{\rho 0}^{2}$ ) and $z_{B}$ versus horizontal imposed magnetic field strength, $B_{0} / B_{\text {eq }}$. The maximum growth rates for $\gamma=5 / 3$
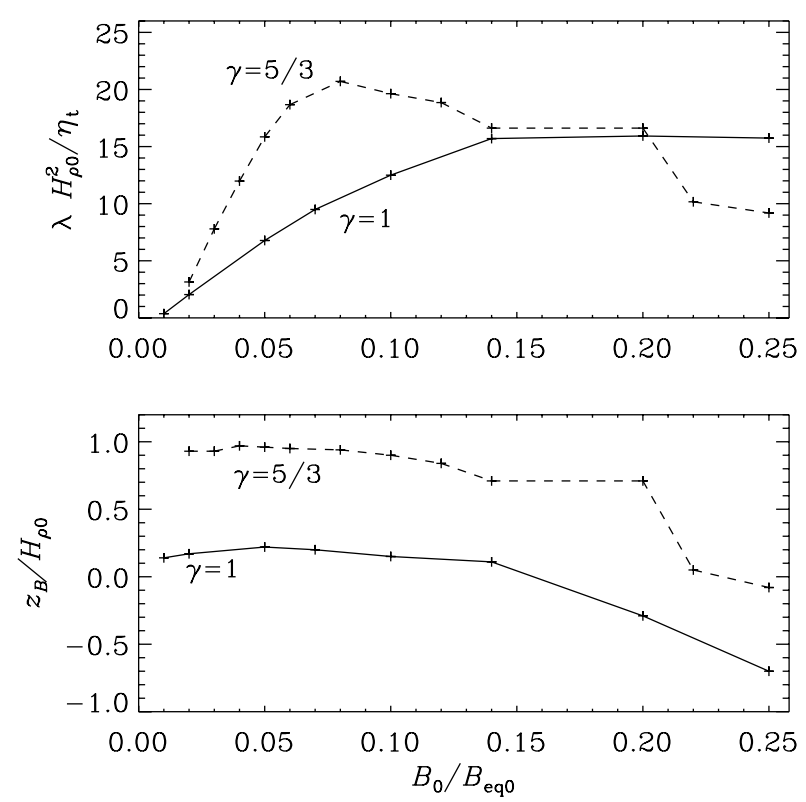

Fig. 11. Same as Fig. 10 (horizontal field), but for Model II.
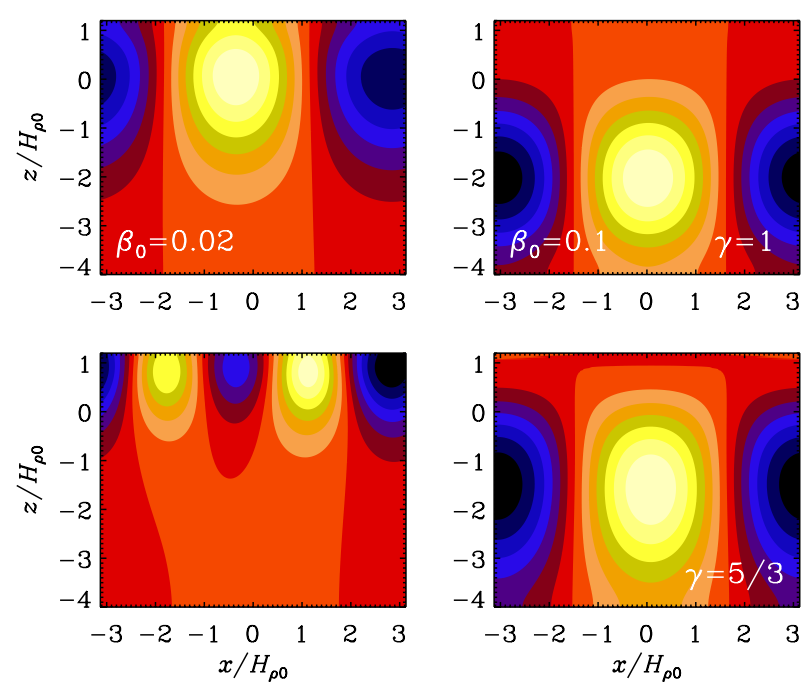

Fig. 12. Snapshots of $\bar{B}_{y}$ from MFS during the kinematic growth phase for different values of $\gamma$ and $B_{0} / B_{\text {eq } 0}$ for $\gamma=1$ (top) and $5 / 3$ (bottom) with $\beta_{0}=0.02$ (left) and 0.1 (right) in the presence of a horizontal field for Model I.

and $\gamma=1$ are similar for both models $\left(4-5 \eta_{\mathrm{t}} / H_{\rho 0}^{2}\right.$ for Model I and $16-20 \eta_{\mathrm{t}} / H_{\rho 0}^{2}$ for Model II). It turns out that for $\gamma=5 / 3$, the growth rate $\lambda$ attains a maximum at some value $B_{0}=B_{\max }$, and then it decreases with increasing $B_{0}$, while in an isothermal run $\lambda$ is nearly constant for greater field strength, except near the surface where the proximity to the boundary is too small. This close proximity reduces the growth rate. For Model I with $\gamma=1$, the decline of $\lambda$ (toward weaker fields on the lefthand side of the plot) begins when the distance to the top boundary ( $z_{\text {top }}-z_{B} \approx 1.2 H_{\rho 0}$ for $\beta_{0}=0.02$ ) is less than the radius of magnetic structures $\left(R \approx 2 \pi / k_{\perp} \approx 1.5 H_{\rho 0}\right.$ using $\left.k_{\perp} H_{\rho 0}=1\right)$. In Model II with $\gamma=1$, the decline of $\lambda$ occurs for stronger fields, but the distance to the top boundary $\left(\approx 1.0 H_{\rho 0}\right)$ is still nearly the same as for Model I.

In an isothermal layer, the height where the eigenfunction peaks is known to decrease with increasing field strength; see 
I. R. Losada et al.: Magnetic flux concentrations in a polytropic atmosphere
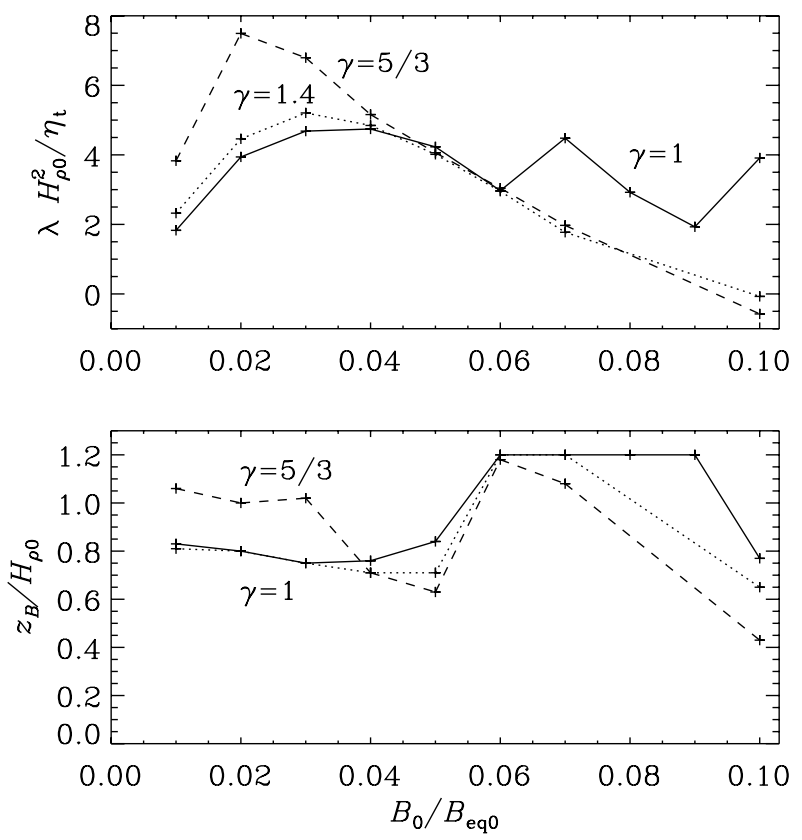

Fig. 13. Dependence of growth rate and optimal depth of NEMPI on field strength from MFS for different values of $\gamma$ in the presence of a vertical field for Model I.

Fig. 6 of Kemel et al. (2012a). One might have expected this decrease to be less steep in the polytropic case, because the optimal depth where NEMPI occurs cannot easily be decreased without suffering a dramatic decrease of the growth rate. This is however not the case, and we find that the optimal depth of NEMPI is now falling off more quickly in Model I, but is more similar for Model II; see the second panels of Figs. 10 and 11. This means that in a polytropic layer, NEMPI works more effectively, and its growth rate is fastest when the magnetic field is not too strong. At the same time, the optimal depth of NEMPI increases, i.e., the resulting value of $z_{B}$ increases as $B_{0}$ decreases.

The resulting growth rates are somewhat less for Model I and somewhat higher for Model II than those of earlier meanfield calculations of Kemel et al. (2012a, their Fig. 6), who found $\lambda H_{\mathrm{p} 0}^{2} / \eta_{\mathrm{t}} \approx 9.7$ in a model with $\beta_{\star}=0.32$ and $\beta_{\mathrm{p}}=0.05$. These differences in the growth rates are plausibly explained by differences in the mean-field parameters.

Visualizations of the resulting horizontal field structures are shown in Fig. 12 for two values of $\gamma$ and $B_{0}$. Increase in the parameter $\gamma$ results in a stronger localization of the instability at the surface layer, where the density scale height is minimum and the growth of NEMPI is strongest.

\subsection{Vertical fields}

In the presence of a vertical field, the early evolution of the instability is similar to that for a horizontal field. In both cases, the maximum field strength occurs at a somewhat larger depth when saturation is reached, except that shortly before saturation there is a brief interval during which the location of maximum field strength rises slightly upwards in the vertical field case. In the saturated case, however, the flux concentrations from NEMPI are much stronger compared to the case of a horizontal field and it leads to the formation of magnetic flux concentrations of equipartition field strength (Brandenburg et al. 2013, 2014). This is possible because the resulting vertical flux tube is not advected downward with the flow that develops as a
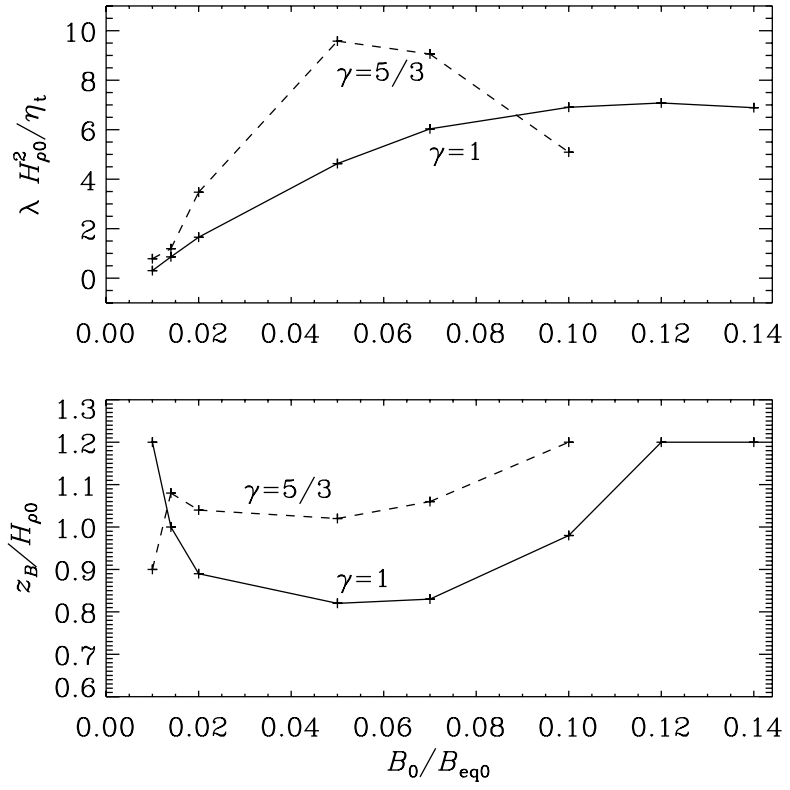

Fig. 14. Same as Fig. 13 (vertical field), but for Model II.

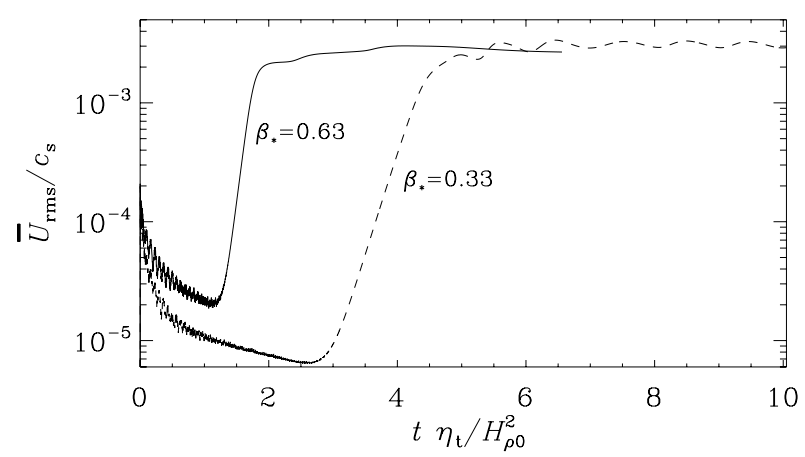

Fig. 15. Comparison of $\bar{U}_{\text {rms }}$ from MFS for Models I (solid line) and II (dashed line), showing exponential growth followed by nonlinear saturation. In both cases we have $\gamma=5 / 3$ and an imposed vertical magnetic field with $\beta_{0}=0.05$.

consequence of NEMPI. The latter effect is the aforementioned "potato-sack" effect, which acts as a nonlinear saturation mechanism of NEMPI with a horizontal field.

In Figs. 13 and 14 we plot the growth rates $\lambda$ and the heights where the eigenfunction attains its maximum values for different $\beta_{0}=B_{0} / B_{\text {eq0 }}$ for Models I and II, respectively. For $\gamma=5 / 3$, the maximum growth rate is higher larger than for $\gamma=1$. This is true for Models I and II, where they are $8-10 \eta_{\mathrm{t}} / H_{\rho 0}^{2}$ for $\gamma=5 / 3$ and $5-7 \eta_{\mathrm{t}} / H_{\rho 0}^{2}$ for $\gamma=1$. The nonmonotonous behavior seen in the dependence of $\lambda$ on $B_{0}$ is suggestive of the presence of different mode structures, although a direct inspection of the resulting magnetic field did not show any obvious differences. However, this irregular behavior may be related to artifacts resulting from a finite domain size and were not regarded important enough to justify further investigation.

Next we focus on a comparison of the growth rates obtained from MFS for horizontal and vertical fields. The values of $\beta_{0}, z_{B}$, and $\beta\left(z_{B}\right)=B_{0} / B_{\text {eq }}\left(z_{B}\right)$ for horizontal and vertical fields are compared in Table 1 for both models. We see that NEMPI is most effective in regions where the mean magnetic field is a small fraction of the local equipartition field and typically slightly less for $\gamma=5 / 3$ than for $\gamma=1$. Indeed, for 

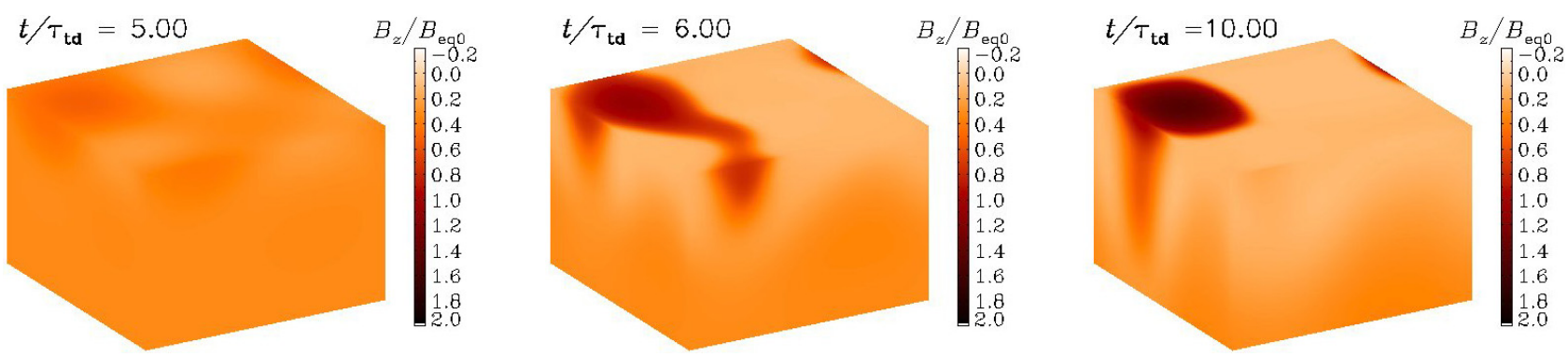

Fig. 16. Snapshots from MFS showing $\bar{B}_{z}$ on the periphery of the computational domain for $\gamma=5 / 3$ and $\beta_{0}=0.05$ at different times for Model I for the case of a vertical field.
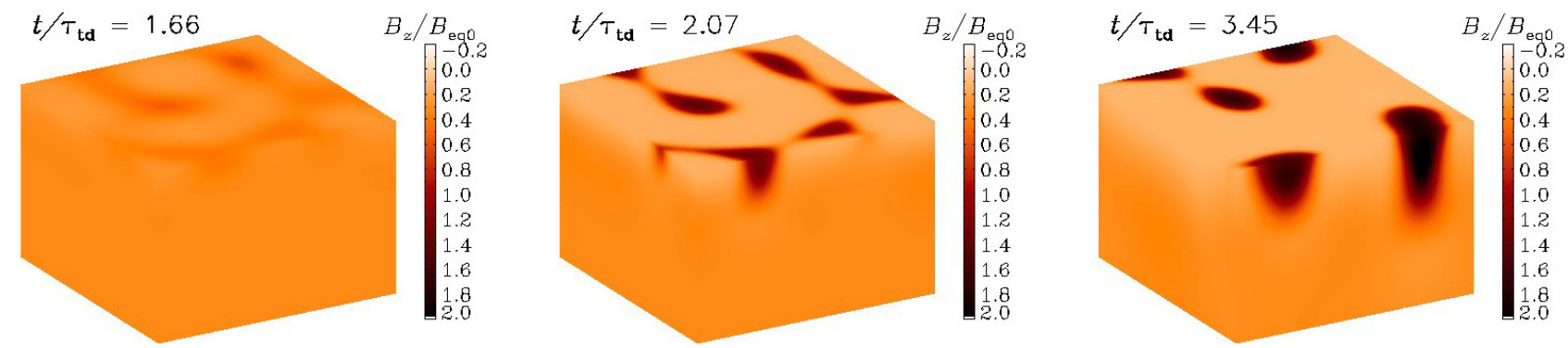

Fig. 17. Similar to Fig. 16 of MFS, but for Model II at times similar to those in the DNS of Fig. 5. There are now more structures than in the earlier MFS of Fig. 16, and they develop more rapidly.

Table 1. Comparison of the optimal depth $z_{B}$ and the corresponding normalized magnetic field strength $\beta\left(z_{B}\right)$ for three values of $\gamma$ for imposed horizontal and vertical magnetic fields of normalized strengths $\beta_{0}=B_{0} / B_{\text {eq } 0}$, for Model I.

\begin{tabular}{cc|ccc|ccc}
\hline \hline & & \multicolumn{3}{|c|}{ Horizontal field } & \multicolumn{3}{c}{ Vertical field } \\
Mod & $\gamma$ & $\beta_{0}$ & $z_{B} / H_{\rho 0}$ & $\beta\left(z_{B}\right)$ & $\beta_{0}$ & $z_{B} / H_{\rho 0}$ & $\beta\left(z_{B}\right)$ \\
\hline I & 1 & 0.05 & -0.76 & 0.034 & 0.04 & 0.76 & 0.058 \\
I & 1.4 & 0.03 & 0.34 & 0.035 & 0.03 & 0.75 & 0.043 \\
I & $5 / 3$ & 0.02 & 0.85 & 0.029 & 0.02 & 1.0 & 0.031 \\
\hline II & 1 & 0.20 & -0.29 & 0.17 & 0.12 & 1.2 & 0.22 \\
II & $5 / 3$ & 0.08 & 0.94 & 0.12 & 0.05 & 1.0 & 0.08 \\
\hline
\end{tabular}

Model I, $\beta\left(z_{B}\right)$ is $3-4 \%$ for horizontal fields and $3-6 \%$ for vertical fields, while for Model II, $\beta\left(z_{B}\right)$ is $12-17 \%$ for horizontal fields and $8-22 \%$ for vertical fields. Here, we have used $\beta\left(z_{B}\right)=\beta_{0} e_{2-\gamma}\left(z_{B} / 2 H_{\rho 0}\right)$, where $e_{q}(x)$ is the $q$-exponential function defined by Eq. (3).

We expect that higher values of $\beta \star$ will lead to greater growth rates. To verify this, we compare in Fig. 15 the time evolutions of $\bar{U}_{\text {rms }}$ for Models I (with $\beta_{\star}=0.33$ ) and Model II (with $\left.\beta_{\star}=0.63\right)$. The growth rate has now increased by a factor of 2.4 (from $\lambda H_{\rho 0}^{2} / \eta_{\mathrm{t}}=3.9$ to 9.5 ), which is slightly more than what is expected from $\beta_{\star}$, which has increased by a factor of 1.9. This change in the growth rate can also be seen in Fig. 11 and Fig. 14 for Model II (in comparison with Figs. 10 and 13 for Model I). The dependence of the growth rate on the magnetic field strength is qualitatively similar for Models I and II. In particular, it becomes constant for $\gamma=1$, but declines for $\gamma=5 / 3$ as the field increases. The increase of $z_{B}$ with $B_{0}$ is, however, less strong for Model II.

Snapshots of $\bar{B}_{z}$ from MFS for $\gamma=5 / 3$ and $\beta_{0}=0.05$ at different times for Model I are shown in Fig. 16. Comparison with the results of MFS for Model II (see Fig. 17) shows that
Model II fits the DNS better. This is also seen by comparing Fig. 17 with Fig. 5. However, our basic conclusions formulated in this paper are not affected.

\section{Conclusions}

The present work has demonstrated that in a polytropic layer, both in MFS and DNS, NEMPI develops primarily in the uppermost layers, provided the mean magnetic field is not too strong. If the field gets stronger, NEMPI can still develop, but the magnetic structures now occur at greater depths and the growth rate of NEMPI is lower. However, at some point when the magnetic field gets too strong, NEMPI is suppressed in the case of a polytropic layer, while it would still operate in the isothermal case, provided the domain is deep enough. The slow down of NEMPI is not directly a consequence of a longer turnover time at greater depths, but it is related to stratification being too weak for NEMPI to be excited.

By and large, the scaling relations determined previously for isothermal layers with constant scale height still seem to apply locally to polytropic layers with variable scale heights. In particular, the horizontal scale of structures was previously determined to be about 6-8 $H_{\rho}$ (Kemel et al. 2013; Brandenburg et al. 2014). Looking now at Fig. 4 , we see that for $\beta_{0}=0.02$ and $\gamma=5 / 3$, the structures have a wavelength of $\approx 3 H_{\rho 0}$, but this is at a depth where $H_{\rho} \approx 0.3 H_{\rho 0}$. Thus, locally we have a wavelength of $\approx 10 H_{\rho}$. The situation is similar in the next panel of Fig. 4 where the wavelength is $\approx 6 H_{\rho 0}$, and the structures are at a depth where $H_{\rho} \approx 1.5 H_{\rho 0}$, so locally we have a wavelength of $\approx 9 H_{\rho}$. We can therefore conclude that our earlier results for isothermal layers can still be applied locally to polytropic layers.

A new aspect, however, that was not yet anticipated at the time, concerns the importance of NEMPI for vertical fields. While NEMPI with horizontal magnetic field still leads to downflows in the nonlinear regime (the "potato-sack" effect), our present work now confirms that structures consisting of vertical 
fields do not sink, but reach a strength comparable to or in excess of the equipartition value (Brandenburg et al. 2013, 2014). This makes NEMPI a viable mechanism for spontaneously producing magnetic spots in the surface layers. Our present study therefore supports ideas about a shallow origin for active regions and sunspots (Brandenburg 2005; Brandenburg et al. 2010; Kitiashvili et al. 2010; Stein \& Nordlund 2012), contrary to common thinking that sunspots form near the bottom of the convective zone (Parker 1975; Spiegel \& Weiss 1980; D'Silva \& Choudhuri 1993). More specifically, the studies of Losada et al. (2013) point toward the possibility that magnetic flux concentrations form in the top $6 \mathrm{Mm}$, i.e., in the upper part of the supergranulation layer.

There are obviously many other issues of NEMPI that need to be understood before it can be applied in a meaningful way to the formation of active regions and sunspots. One question is whether the hydrogen ionization layer and the resulting $\mathrm{H}^{-}$opacity in the upper layers of the Sun are important in providing a sharp temperature drop and whether this would enhance the growth rate of NEMPI, just like strong density stratification does. Another important question concerns the relevance of a radiating surface, which also enhances the density contrast. Finally, of course, one needs to verify that the assumption of forced turbulence is useful in representing stellar convection. Many groups have considered magnetic flux concentrations using realistic turbulent convection (Kitiashvili et al. 2010; Rempel 2011; Stein \& Nordlund 2012). However, only at sufficiently large resolution can one expect strong enough scale separation between the scale of the smallest eddies and the size of magnetic structures. That is why forced turbulence has an advantage over convection. Ultimately, however, such assumptions should no longer be necessary. On the other hand, if scale separation is poor, our present parameterization might no longer be accurate enough, and one would need to replace the multiplication between $q_{\mathrm{p}}$ and $\overline{\boldsymbol{B}}^{2}$ in Eq. (25) by a convolution. This possibility is fairly speculative and requires a separate investigation. Nevertheless, in spite of these issues, it is important to emphasize that the qualitative agreement between DNS and MFS is already surprisingly good.

Acknowledgements. This work was supported in part by the European Research Council under the AstroDyn Research Project No. 227952, by the Swedish Research Council under the project grants 621-2011-5076 and 2012-5797 (I.R.L., A.B.), by EU COST Action MP0806, by the European Research Council under the Atmospheric Research Project No. 227915, and by a grant from the Government of the Russian Federation under contract No. 11.G34.31.0048 (N.K., I.R.). We acknowledge the allocation of computing resources provided by the Swedish National Allocations Committee at the Center for Parallel Computers at the Royal Institute of Technology in Stockholm and the National
Supercomputer Centers in Linköping, the High Performance Computing Center North in Umeå, and the Nordic High Performance Computing Center in Reykjavik.

\section{References}

Brandenburg, A. 2005, ApJ, 625, 539

Brandenburg, A., Kleeorin, N., \& Rogachevskii, I. 2010, Astron. Nachr., 331, 5

Brandenburg, A., Kemel, K., Kleeorin, N., Mitra, D., \& Rogachevskii, I. 2011 ApJ, 740, L50

Brandenburg, A., Kemel, K., Kleeorin, N., \& Rogachevskii, I. 2012, ApJ, 749, 179

Brandenburg, A., Kleeorin, N., \& Rogachevskii, I. 2013, ApJ, 776, L23

Brandenburg, A., Gressel, O., Jabbari, S., Kleeorin, N., \& Rogachevskii, I. 2014, A\&A, 562, A53

D’Silva, S., \& Choudhuri, A. R. 1993, A\&A, 272, 621

Jabbari, S., Brandenburg, A., Kleeorin, N., Mitra, D., \& Rogachevskii, I. 2013, A\&A, 556, A106

Käpylä, P. J., Brandenburg, A., Kleeorin, N., Mantere, M. J., \& Rogachevskii, I. 2012, MNRAS, 422, 2465

Kemel, K., Brandenburg, A., Kleeorin, N., \& Rogachevskii, I. 2012a, Astron. Nachr., 333, 95

Kemel, K., Brandenburg, A., Kleeorin, N., Mitra, D., \& Rogachevskii, I. 2012b, Sol. Phys., 280, 321

Kemel, K., Brandenburg, A., Kleeorin, N., Mitra, D., \& Rogachevskii, I. 2013, Sol. Phys., 287, 293

Kitiashvili, I. N., Kosovichev, A. G., Wray, A. A., \& Mansour, N. N. 2010, ApJ, 719,307

Kleeorin, N., \& Rogachevskii, I. 1994, Phys. Rev. E, 50, 2716

Kleeorin, N. I., Rogachevskii, I. V., \& Ruzmaikin, A. A. 1989, Sov. Astron. Lett., 15,274

Kleeorin, N. I., Rogachevskii, I. V., \& Ruzmaikin, A. A. 1990, Sov. Phys. JETP, 70,878

Kleeorin, N., Mond, M., \& Rogachevskii, I. 1993, Phys. Fluids B, 5, 4128

Kleeorin, N., Mond, M., \& Rogachevskii, I. 1996, A\&A, 307, 293

Krause, F., \& Rädler, K.-H. 1980, Mean-field Magnetohydrodynamics and Dynamo Theory (Oxford: Pergamon Press)

Losada, I. R., Brandenburg, A., Kleeorin, N., Mitra, D., \& Rogachevskii, I. 2012, A\&A, 548, A49

Losada, I. R., Brandenburg, A., Kleeorin, N., \& Rogachevskii, I. 2013, A\&A, $556, \mathrm{~A} 83$

Newcomb, W. A. 1961, Phys. Fluids, 4, 391

Parker, E. N. 1955, ApJ, 121, 491

Parker, E. N. 1966, ApJ, 145, 811

Parker, E. N. 1975, ApJ, 198, 205

Plastino, A. R., \& Plastino, A. 1993, Phys. Lett. A, 174, 384

Rempel, M. 2011, ApJ, 740, 15

Rogachevskii, I., \& Kleeorin, N. 2007, Phys. Rev. E, 76, 056307

Spiegel, E. A., \& Weiss, N. O. 1980, Nature, 287, 616

Spruit, H. C. 1981, A\&A, 98, 155

Schüssler, M., Caligari, P., Ferriz-Mas, A., \& Moreno-Insertis, F. 1994, A\&A, 281, L69

Stein, R. F., \& Nordlund, ̊̊. 2012, ApJ, 753, L13

Sur, S., Brandenburg, A., \& Subramanian, K. 2008, MNRAS, 385, L15

Tsallis, C. 1988, J. Stat. Phys., 52, 479

Vitense, E. 1953, Z. Astrophys., 32, 135

Yamano, T. 2002, Stat. Mech. Appl., 305, 486

Warnecke, J., Losada, I. R., Brandenburg, A., Kleeorin, N., \& Rogachevskii, I. 2013, ApJ, 777, L37 